\title{
Two Algorithms for Detection of Mutually Occluding Traffic Signs
}

\author{
Thanh Bui-Minh, Ovidiu Ghita, Paul F. Whelan, Senior Member, IEEE, Trang Hoang, Vinh Quang Truong
}

\begin{abstract}
The robust identification of the traffic signs represents the first and one of the most important steps in the development of a traffic sign recognition (TSR) system. Traffic signs detection usually involves a color segmentation process that uses the information related to the chromatic properties of the road signs. Since the traffic video data is captured in diverse road and weather conditions, the problem relating to traffic sign detection is quite challenging. Among several issues that need to be addressed during this processing stage, the problem generated by mutually occluding traffic signs (mutual occlusion occurs when one traffic sign partially occludes the surface of other road signs) that are attached to the same pole require special attention. In these situations the color segmentation process fails to correctly identify the regions that are associated with the traffic signs. These traffic sign detection failures compromise the performance of other stages of the TSR system and in this paper we propose two approaches that address the segmentation of mutually occluding traffic signs. The first approach uses the information associated with the inner parts of the traffic signs, while the second approach applies the watershed transform to identify the signs that have their borders in contact or are mutually occluding.
\end{abstract}

Index Terms-Traffic signs, Color segmentation, Mutual occlusion, Watershed.

\section{INTRODUCTION}

$\mathrm{T}$ raffic signs recognition (TSR) has been a challenging problem that started to be investigated since 1980 , and recently it has received significant interest from companies that develop automotive safety equipment [12]. At present, several TSR systems that primarily aim at the detection and recognition of speed limit signs in traffic video data are commercially available, and they have been already deployed in the development of the safety equipment for medium and high-end cars. (Commercial TSR systems have been developed by Mobileye, Continental AG, Siemens and it is worth noting that these systems in addition to TSR also include other features such as Lane Departure Warning and Intelligent Headlight Control $[13,14])$. The recent TSR systems represent a welcome addition, as they are able to provide the drivers with correct information about an important category of traffic signs, which will have an important role in preventing accidents, saving lives and increasing the driving

Ovidiu Ghita and Paul F. Whelan are with the School of Electronic Engineering, Dublin City University, Dublin, Ireland (e-mail: ghitao@eeng.dcu.ie, paul.whelan@dcu.ie).

Thanh Bui-Minh and Truong Quang Vinh are with the Hochiminh City University of Technology, Hochiminh City, Vietnam (e-mail: thanh.buiminh2@mail.dcu.ie, tqvinh@hcmut.edu.vn). performance especially in situations when the road signs are partially occluded, damaged or placed in unexpected locations. However, since the current range of commercial TSR systems is able to provide information to the driver only for a very limited range of traffic signs (most recent TSR systems have been extended to alert the driver not only about speed limits but also about overtaking restrictions), additional work is required to extend the functionality of the current TSR systems by including more categories of traffic signs and to improve the robustness of the traffic sign classification in the presence of difficult road and weather conditions $[4,10,12]$.

TSR involves two principal stages: traffic sign detection and traffic sign classification or recognition. In the first stage, the image is pre-processed and segmented according to several properties such as color or shape that are characteristic for each type of traffic sign. The output of this initial process is a segmented image where potential regions that may be associated with road signs are highlighted. The efficiency and the speed of the traffic sign detection are critically important factors, as the output generated by this stage is subsequently used for traffic sign recognition. In the recognition stage, the traffic sign candidates are verified with respect to a certain set of features in order to decide if the candidates are road signs or background objects, and to classify them in specific traffic sign categories (prohibitory, mandatory, warning, stop and yield, etc.).

Traffic sign video frames are captured from a moving vehicle and they present several distortions that are caused by intensity inconsistencies due to various illumination conditions, shadows, rain, etc. Additional problems are generated by background objects that resemble the color characteristics of particular traffic signs. This challenging road environment complicates the traffic sign detection and several algorithms have been proposed to address this problem $[1,2,3,4,5,7,8,10,11]$. Most of the developed TSR algorithms employed specific features relating to the shape and color that are extracted from different categories of road signs. Thus, Escalera et al [8] proposed a two-step implementation, where in the first step the candidate locations in the video frames (that are consistent with the color schemes that are specific for traffic signs) are identified. Then, in the second stage, a multilayer perceptron neural network classifier is applied to decide if the candidate traffic signs were correctly identified and to evaluate the response of the classifier with respect to each traffic sign sample included in the training set. A related twostep implementation was proposed by Maldonado-Bascó et al 
[1,3]. The first step of their TSR algorithm dealt with the detection of the road sign candidates using linear SVMs, while the second stage addressed the traffic sign recognition using Gaussian-kernel SVMs. This line of approach (that involves the two-step analysis of traffic sign images) was followed in other TSR implementations [see for example 1-4 and 7-12], and in these works the main focus was on the development of optimal strategies that use color and shape information for accurate traffic sign recognition. Since these works on TSR mainly addressed several difficult problems relating to feature extraction and the development of complex traffic sign recognition algorithms, the problem associated with the identification of the mutually occluding traffic signs (and traffic signs for which their outlines are in contact) that are attached to the same pole has received limited attention. Mutual occlusion occurs when multiple traffic signs show different degrees of occlusion (or overlap) when they are attached to the same pole. The incidence of the mutual traffic sign occlusion generates errors in traffic sign detection, as only one object is detected for all partly occluded traffic signs. The identification of only one object that corresponds to all mutually occluding traffic signs compromises the accuracy of the TSR, as this candidate region is substantially different than the image regions that are used to train the traffic sign classifier. Fig. 1 presents an example that illustrates three mutually occluding traffic signs, and the goal of this work is to describe the development of two algorithms that are able to identify the traffic signs in this complex environment.

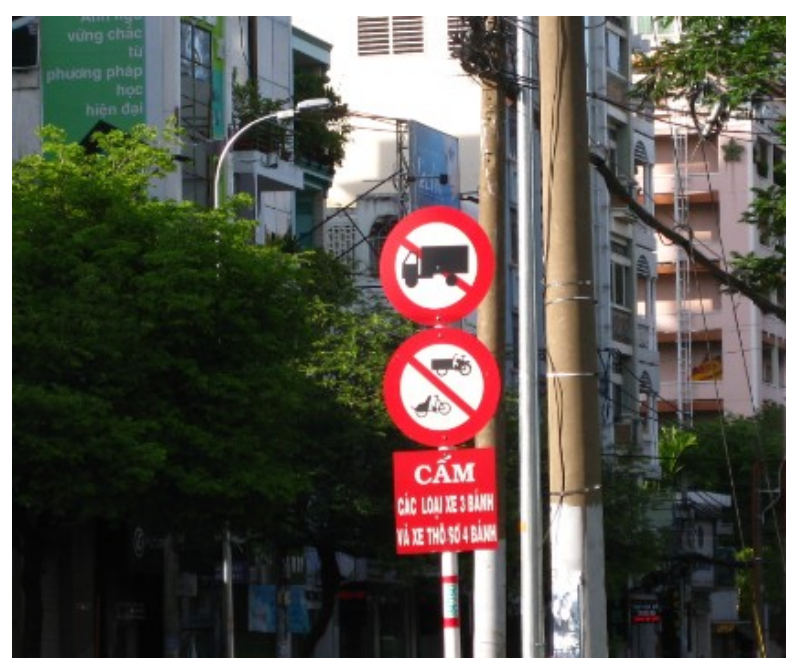

Fig. 1. An example that illustrates three mutually occluding traffic signs.

This paper is organized as follows. Section I presents an introductory discussion about the past works that addressed the development of TSR systems. Section II describes the color segmentation step. Sections III and IV provide details about the two approaches developed for the detection of mutually occluding traffic signs and experimental results are reported in Section V. The paper is ended with several concluding remarks that are presented in Section VI.

\section{COLOR SEgmentation}

The main goal of the color segmentation is to generate a binary image that highlights the candidate regions that may be associated with road signs and to reject the background and the other irrelevant objects. Since the traffic video data is captured in diverse weather conditions, the hue component of the color space played a key role in the development of traffic sign detection algorithms. The main reason for using the hue as input for traffic sign detection is given by the fact that this information shows increased resilience to changes in light conditions and is more robust when dealing with intensity saturation [4]. In our TSR system we have implemented a version of the color segmentation that has been proposed by Escalera et al [8]. We have chosen this method as it returns better accuracy than other traffic sign detection algorithms that were detailed in [2] and [4]. Escalera et al method involves the conversion of the input image from RGB to HSI color space to exploit the increased invariance of the Hue component and specific threshold conditions are imposed to determine the regions in the image that are predominantly red and blue. Fig. 2 presents an example that illustrates the output returned by the Escalera et al color segmentation algorithm. While the predominantly red regions are correctly detected in the output returned by the color segmentation algorithm, it can also be observed that the red regions that belong to the two traffic signs generate a connected object in the binary image. Since the next stage of the algorithm generates a bounding rectangle for all connected components that are present in the binary image, the region that is generated by the two road signs is not suitable for traffic sign classification. Thus, before traffic sign classification, an additional process has to be applied to detach the two connected red rims and generate individual candidate regions for both of them.

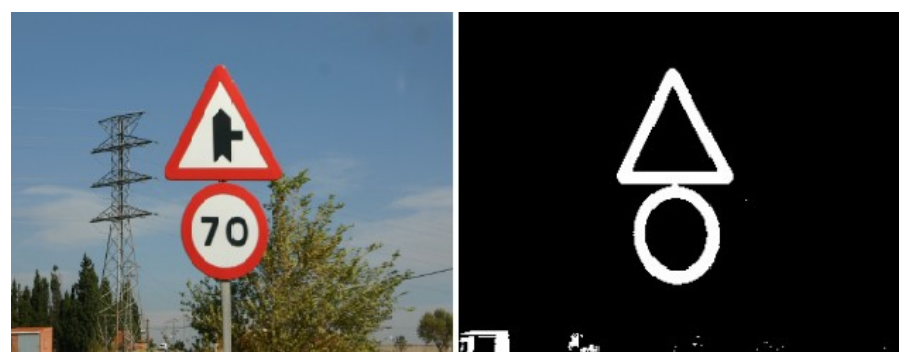

Fig. 2. An example illustrating the red color segmentation for two mutually occluding traffic signs that are attached to the same pole.

III. GENERATION OF CANDIDATE REGIONS FOR MUTUALLYOCCLUDING TRAFFIC SIGNS USING THE SIGNS' INNER SECTIONS

As indicated in Fig. 2, a difficult problem is given by situations when multiple traffic signs with the same rim color are attached to a common pole and they present mutual occlusions. In these cases the color segmentation method detailed in the previous section will generate a single region for all traffic signs that present any degree of overlap (or occlusion). 

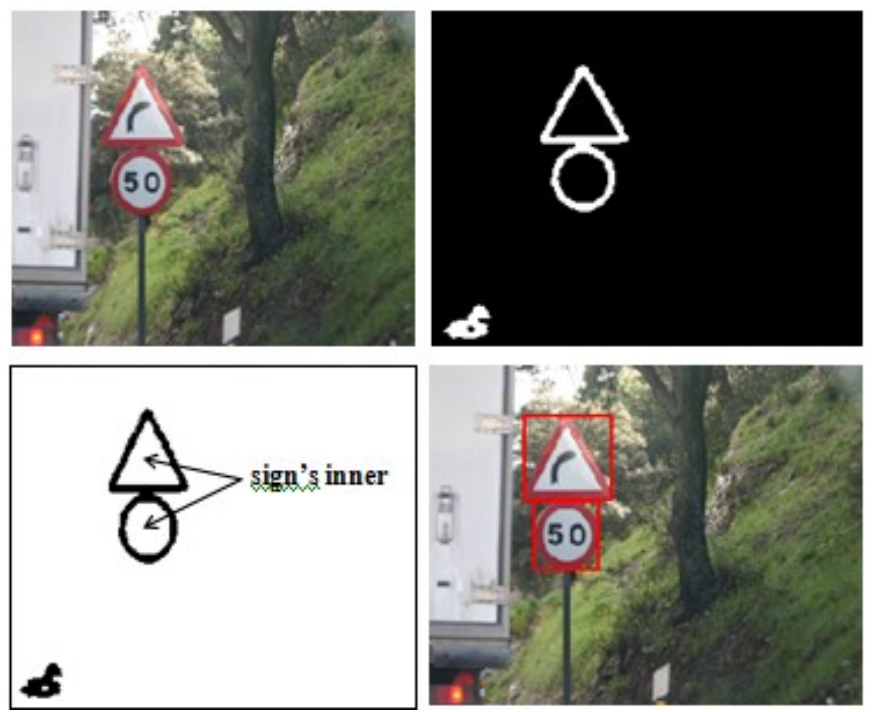

Fig. 3. Identification of the region of interest for overlapped traffic signs. From left to right and top to bottom: input image, color segmentation result, identification of the inner parts of the traffic signs and identification of the region of interest (marked with a red rectangle) for each traffic sign. This figure is best viewed in color.

Fig. 3 illustrates another example where the color segmentation process identifies a single region that encompasses two traffic signs. Since the traffic sign classifiers are trained using sample regions that encompass one traffic sign, the candidate region illustrated in Fig. 3 will fail the classification test. To address this problem, we propose to divide the candidate region into regions that include individual traffic signs. In Fig. 3 we can notice that the outline of the traffic signs is only partially overlapped, and the identification of the traffic signs' rims can be obtained if we locate the inner sections of the traffic signs.

In our approach we employed the information that the inner parts of the traffic signs are painted in bright colors (this is done to emphasize the chromatic contrast with the outer part of the traffic sign). The traffic signs that regulate the Vietnam roads have their inner parts painted in white and the text or graphical symbols are painted in black. Using this knowledge we developed an algorithm to detach the rims of the overlapped traffic which involves a sequence of operations that are detailed in Fig. 4. The input image is smoothed with the median filter to remove the impulse noise. Next, the Escalera et al [8] color segmentation process is applied to identify the candidate regions and the inner sections of the traffic signs are detected by applying the brightness inversion operation. As depicted in Fig. 3, we can observe that the shapes of the inner parts are similar to the shapes of the traffic signs, and most importantly the inner parts of the overlapped traffic signs are disjoint. Thus, the identification of the inner parts of the traffic signs is sufficient to generate the region of interest (or the minimum bounding rectangle (MBR)) for each traffic sign contained in the candidate region. The proposed method initially calculates the coordinates of the minimum bounding rectangles for all detected inner sections, and the MBR for each traffic sign is determined by enlarging the rectangle of the inner part with respect to the thickness of the sign's rim. The main steps of the proposed traffic sign detection algorithm detailed in Fig. 4 are illustrated in Fig. 3(b-d).

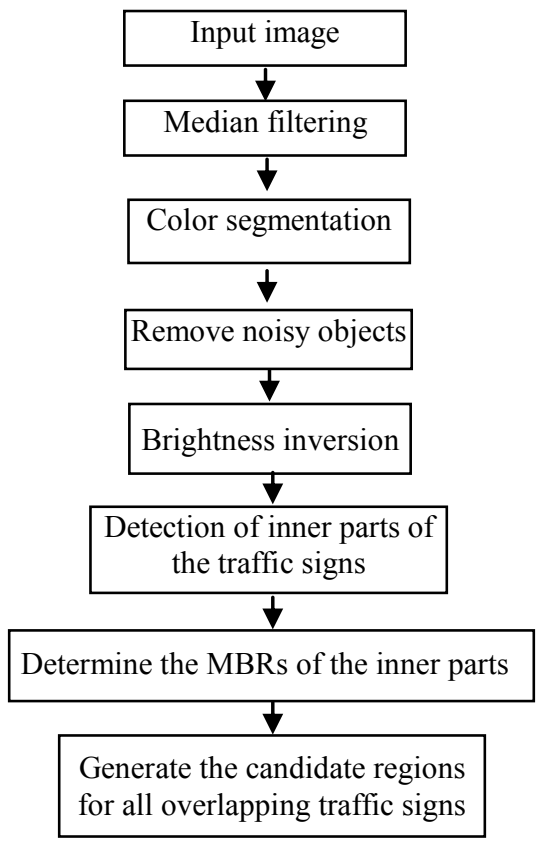

Fig. 4. Generation of the candidate regions for overlapped traffic signs using the signs' inner sections.

The algorithm detailed in Fig. 4 proved efficient under the condition that the level of overlap between traffic signs is not significant. When dealing with significant levels of overlap, the shapes of the inner parts are distorted (when compared to the original shapes of the traffic signs) and the selection of the MBR is incorrect (and results in the generation of unsuitable candidate regions for TSR). This issue motivated us to develop another approach based on the application of the watershed transform.

\section{GENERATION OF CANDIDATE REGIONS FOR MUTUALLY} OCCLUDING TRAFFIC SIGNS USING THE WATERSHED TRANSFORM

Fig. 5 illustrates the overview of the second method that involves watershed segmentation. The first three stages of this new method are similar to the ones developed for the algorithm detailed in Fig. 4. In this new method, the candidate regions returned by color segmentation are filled to generate objects with uniform properties. In the next step, the distance transform for filled candidate regions is computed using the Euclidean distance and this information is further processed using the watershed transform.

The watershed segmentation is an extremely useful technique that is able to divide regions that are generated by overlapped objects. The main issue associated with the use of watershed transform is the identification of the local minima in the distance map, which is required to guide the segmentation 
process. An efficient implementation of this segmentation approach involves the sorting of values in the distance map and the generation of disjoints regions using marker-controlled flood-fill operations. The candidate traffic signs are detached by computing the logical AND operation between the watershed lines and the filled candidate blobs (see Fig. 6). The last step validates the disjoint candidate traffic sign regions with respect to shape and size constraints to ensure that only plausible regions will be evaluated by the traffic sign classification process. Fig. 5 presents an outline of the proposed traffic sign detection method that applies the immersion-based watershed segmentation [5] that is applied to divide the candidate regions that are generated by overlapped traffic signs.

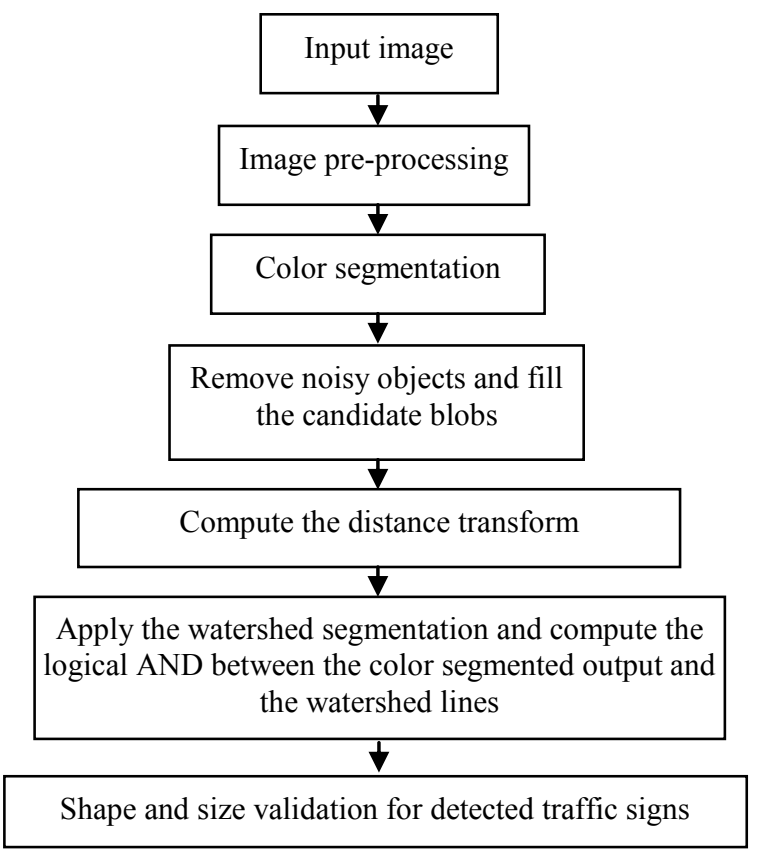

Fig. 5. Generation of the candidate regions for overlapped traffic signs using watershed segmentation.

\section{EXPERIMENTAL RESULTS}

More than 160 traffic images that present overlapped traffic signs that were captured in different environmental conditions have been used to evaluate the robustness of the traffic sign detection methods. The two approaches proved successful when applied to these challenging images, and the successful detection rates are $87.5 \%$ (method that generates the candidate regions using the inner parts of the traffic signs) and $96.8 \%$ (method that generates the candidate regions using watershed segmentation). As indicated in Section III, the method that evaluates the inner parts of the traffic signs returns incorrect results when dealing with large levels of overlaps between mutually occluding traffic signs. The experimental results indicate that the method based on watershed segmentation proved more accurate in these situations (see the additional results presented in Figs. 7 and 8) and for this reason we have included it in the development of the TSR system.
A video sequence that shows the functionality of our TSR system can be found on www.youtube.com by visiting the following link:

\section{http://www.youtube.com/watch?v=zEtpzw4Vtlw}
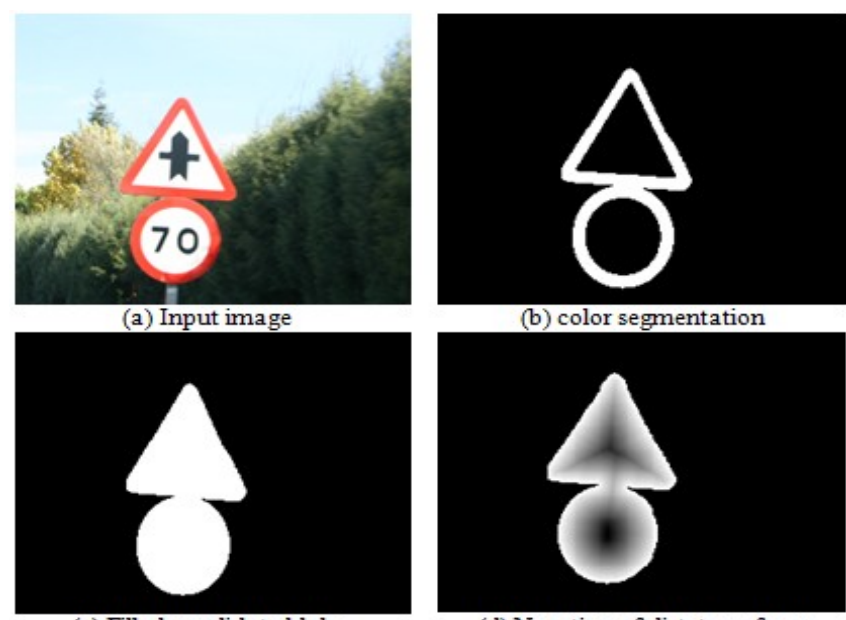

(b) color segmentation

(c) Filled candidate blobs
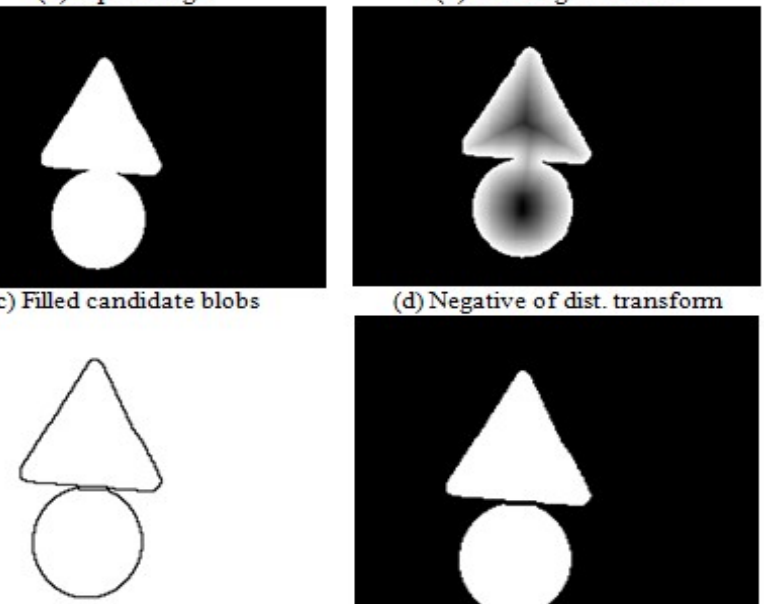

(e) Watershed lines

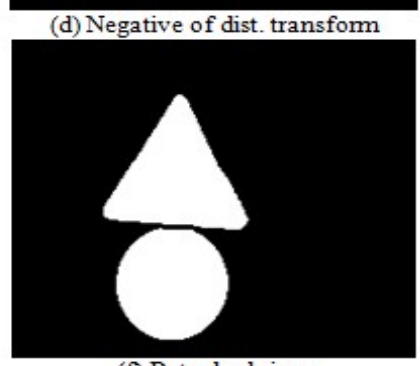

(f) Detached signs

Fig. 6. Generation of the candidate regions for mutually occluding traffic signs using watershed segmentation.

\section{CONCLUSIONS}

This paper detailed the development of two traffic sign detection methods that were developed for the detection of overlapped traffic signs that are attached to the same pole. The experimental results show that the method based on the use of the watershed transform provides better results and this approach has been adopted in the development of the TSR system.

In our future research we will focus on improving the color segmentation process and we will address the implementation of robust traffic sign tracking strategies that will be used to enhance the confidence of the traffic sign recognition process in consecutive frames of the traffic video data.

\section{REFERENCES}

[1] S. Maldonado-Bascón, S. Lafuente-Arroyo, P. Gil-Jiménez, H. GómezMoreno, and F. López-Ferreras, "Road-sign detection and recognition based on Support Vector Machines", IEEE Trans. on Intelligent Transportation Systems, vol. 8, no. 2, pp. 264- 278, 2007.

[2] H. Fleyeh, "Shadow and highlight invariant colour segmentation algorithm for traffic signs", IEEE Conference on Cybernetics and Intelligent Systems, Bangkok, Thailand, June 2006. 


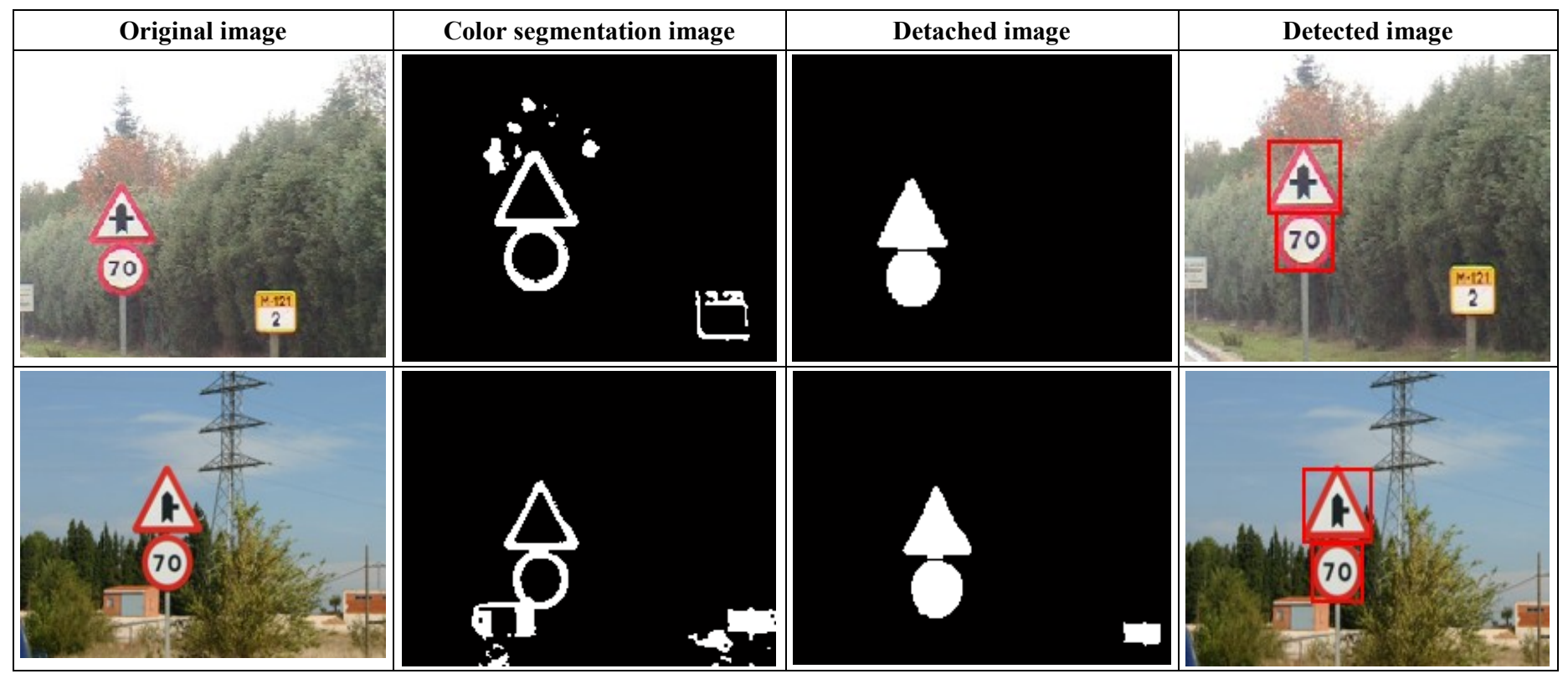

Fig. 7. Examples that illustrate cases where both methods return correct results

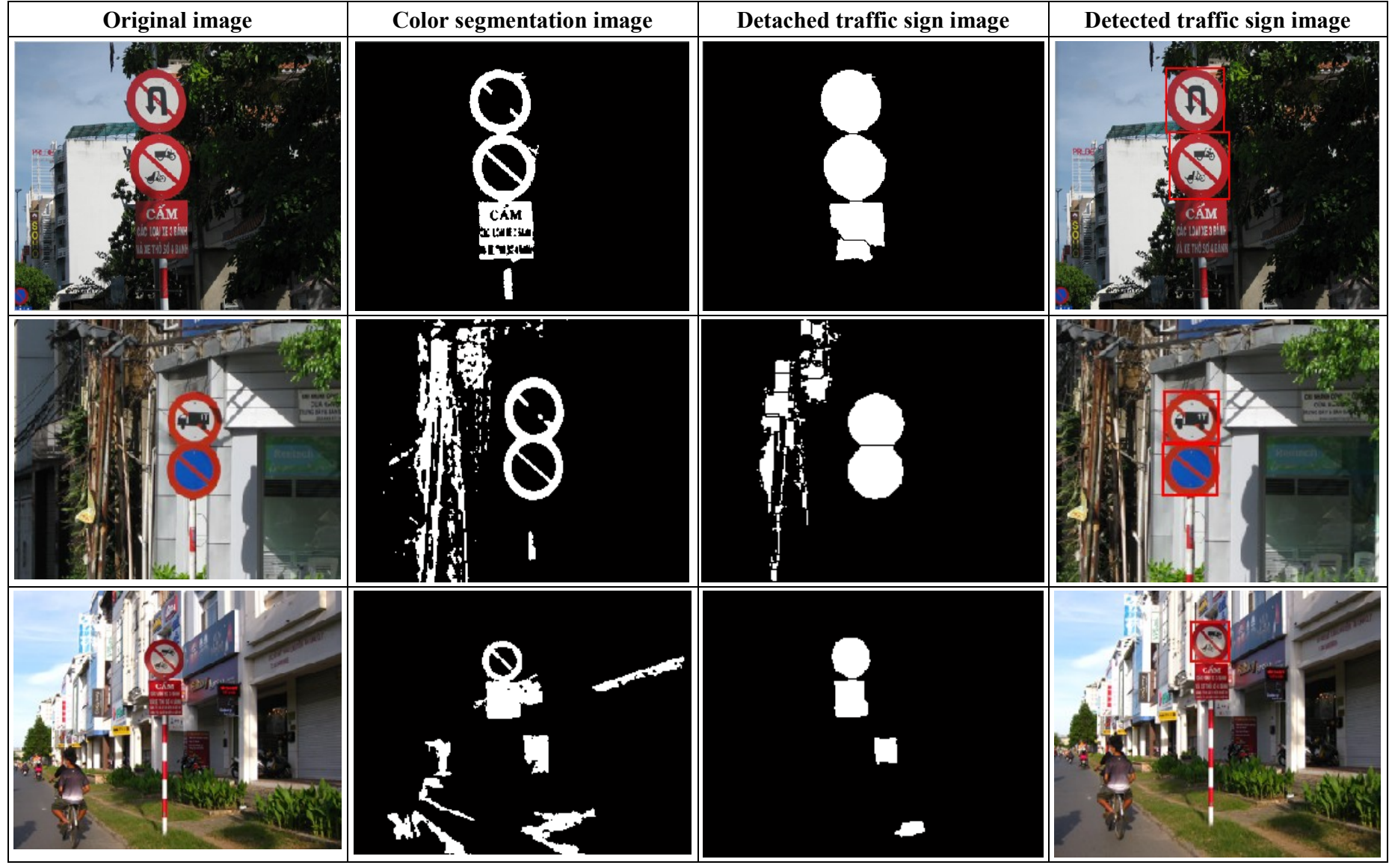

Fig. 8. Examples that illustrate cases where only the method based on watershed segmentation returns correct results. 
[3] S. Maldonado-Bascón, J. Acevedo-Rodríguez, A. Fernández-Caballero, and F. López-Ferreras, "An optimization on pictogram identification for the road-sign recognition task using SVMs", Computer Vision and Image Understanding, vol. 114 , no. 3, pp. 373-383, 2010.

[4] H. Fleyeh, "Traffic and road sign recognition", Dalarna University, Sweden, 2008.

[5] P.F. Whelan and D. Molloy (2000), Machine Vision Algorithms in Java: Techniques and Implementation, Springer (London), 298 Pages. ISBN 185233-218-2.

[6] P. Rosin, "Measuring shape: ellipticity, rectangularity, and triangularity", Machine Vision and Applications, vol. 14, no. 3, pp. 172-184, 2003.

[7] H. Gómez-Moreno, S. Maldonado-Bascón, P. Gil-Jiménez, and S. Lafuente-Arroyo, "Goal evaluation of segmentation algorithms for traffic sign recognition", IEEE Trans. on Intelligent Transportation Systems, vol. 11, no. 4, pp. 917-930, 2010.

[8] A. Escalera, L.E. Moreno, M.A. Salichs, and J. Armingol, "Road traffic sign detection and classification", IEEE Trans. on Industrial Electronics, vol. 44, no. 6, pp. 848-859, 1997.

[9] F. Chang, C.J. Chen, and C.J. Lu, "A linear-time component-labelling algorithm using contour tracing technique", Computer Vision and Image Understanding, vol. 93, no. 2, pp. 206-220, 2004.
[10] M. Meuter, C. Nunn, S. Gormer, S. Muller-Schneiders, and A. Kummert, "A decision fusion and reasoning module for a traffic sign recognition system”, IEEE Trans. on Intelligent Transportation Systems, vol. 12, no. 4, pp. 1126-1134, 2011.

[11] T. Le, S. Tran, S. Mita, and T.D. Nguyen, "Real-time traffic sign detection using color and shape-based features", $2^{\text {nd }}$ Asian Conference on Intelligent Information and Database Systems, LNAI 5991, Hue, Vietnam, 2010.

[12] A. Ruta, Y. Li, and X. Liu, "Real-time traffic sign recognition from video by class-specific discriminative features", Pattern Recognition, vol. 43, no. 1, pp. 416-430, 2010

[13] Mobileye - www.mobileye.com/technology/applications/traffic-signdetection (Accessed - April 30, 2012).

[14] Continental AG - http://www.conti-

online.com/generator/www/de/en/continental/automotive/general/chassis /safety/hidden/verkehrszeichenerkennung_en.html (Accessed - April 30, 2012) 